\title{
Generating All Circular Shifts by Context-Free Grammars in Greibach Normal Form
}

\author{
Peter R.J. Asveld \\ Department of Computer Science, Twente University of Technology \\ P.O. Box 217, 7500 AE Enschede, the Netherlands \\ e-mail: infprja@cs.utwente.nl
}

\begin{abstract}
For each alphabet $\Sigma_{n}=\left\{a_{1}, a_{2}, \ldots, a_{n}\right\}$, linearly ordered by $a_{1}<a_{2}<\cdots<a_{n}$, let $C_{n}$ be the language of circular or cyclic shifts over $\Sigma_{n}$, i.e., $C_{n}=\left\{a_{1} a_{2} \cdots a_{n-1} a_{n}\right.$, $\left.a_{2} a_{3} \cdots a_{n} a_{1}, \ldots, a_{n} a_{1} \cdots a_{n-2} a_{n-1}\right\}$. We study a few families of context-free grammars $G_{n}(n \geq 1)$ in Greibach normal form such that $G_{n}$ generates $C_{n}$. The members of these grammar families are investigated with respect to the following descriptional complexity measures: the number of nonterminals $\nu(n)$, the number of rules $\pi(n)$ and the number of leftmost derivations $\delta(n)$ of $G_{n}$. As in the case of Chomsky normal form, these $\nu, \pi$ and $\delta$ are functions bounded by low-degree polynomials. However, the question whether there exists a family of grammars that is minimal with respect to all these measures remains open.
\end{abstract}

Keywords: context-free grammar, Greibach normal form, permutation, circular shift, cyclic shift, descriptional complexity, unambiguous grammar.

\section{Introduction}

Let $\Sigma_{n}=\left\{a_{1}, a_{2}, \ldots, a_{n}\right\}$ be an alphabet, linearly ordered by $a_{1}<a_{2}<\cdots<a_{n}$, and let $L_{n}$ be the language over $\Sigma_{n}$ of the $n$ ! permutations of $a_{1} a_{2} \cdots a_{n}$. In $2002 \mathrm{G}$. Satta [15] conjectured that "any context-free grammar $G_{n}$ in Chomsky normal form (CNF) that generates $L_{n}$ must have a number of nonterminal symbols that is not bounded by any polynomial function in $n$ ". This statement has been proved in [9], but without showing how to generate $\left\{L_{n}\right\}_{n \geq 1}$ by context-free grammars $\left\{G_{n}\right\}_{n \geq 1}$ in CNF. In [2] we provided several grammar families for $\left\{L_{n}\right\}_{n \geq 1}$ together with the usual descriptional complexity measures as the number of nonterminals $\nu(n)$ and the number of rules $\pi(n)$; cf. $[11,13,14,7,5,1,6]$ for 
these measures. The relative descriptional complexity of these grammar families is anything but straightforward and the quest for a family of minimal grammars (with respect to these complexity measures) remains a challenging problem. Then in [3] we investigated some specific permutations over $\Sigma_{n}$, viz. the circular or cyclic shifts, defined by

$$
C_{n}=\left\{a_{1} a_{2} \cdots a_{n-1} a_{n}, a_{2} a_{3} \cdots a_{n} a_{1}, a_{3} a_{4} \cdots a_{1} a_{2}, \ldots, a_{n} a_{1} \cdots a_{n-2} a_{n-1}\right\} .
$$

An alternative definition of $C_{n}$ in terms of the so-called circular closure operator $c$ on languages $L$, which is defined by $c(L)=\{v u \mid u v \in L\}$ [8], is: $C_{n}=c\left(\left\{a_{1} a_{2} \cdots a_{n}\right\}\right)$.

The fact that the $n$ elements of $C_{n}$ is much less than the $n$ ! elements of $L_{n}$ is also reflected by the complexity measures of the corresponding grammar families: for $\left\{L_{n}\right\}_{n \geq 1}$, the functions $\nu(n)$ and $\pi(n)$ are exponential functions $[15,9,2]$, whereas for $\left\{C_{n}\right\}_{n \geq 1}$, they are bounded by low-degree polynomial functions; cf. [3].

In this paper we investigate a few ways of generating the family $\left\{C_{n}\right\}_{n \geq 1}$ by contextfree grammars in Greibach normal form (GNF) and for these families of grammars we determine the complexity measures $\nu, \pi$ and $\delta$ as functions of $n$.

The results we obtain are rather similar to those in [3], as is the organization of this paper. $\S 2$ is devoted to preliminaries and in $\S 3$ we consider elementary properties of grammars $G_{n}$ in GNF for $C_{n}$. As using the arbitrary GNF for $C_{n}$ is trivial ( $\$ 3$ ), we focus in $\S \S 4-7$ on the Greibach $k$-form $(k=1,2)$. An approach based on the set of circularly ordered strings results in a grammar family in Greibach 2-form ( $\$ 4$ ). Modifying this family into Greibach 1-form in $\S 5$ results in a family with less rules. Unambiguous grammars for $C_{n}$ are studied in $\S 6$ : then $\nu(n)$ and $\pi(n)$ are related in a simple way. In $\S 7$ we discuss minimality for unambiguous grammars in GNF but the existence of a family of grammars for which these complexity measures are minimal remains open. Finally, some concluding remarks are in $\S 8$.

\section{Preliminaries}

For rudiments of discrete mathematics, particularly of combinatorics, and of formal language theory, we refer to standard texts like [10] and [12], respectively.

We denote the empty word by $\lambda$ and the length of the word $x$ by $|x|$. For each word $w$ over $\Sigma, \mathcal{A}(w)$ is defined as the set of all symbols from $\Sigma$ that do occur in $w$. Formally, $\mathcal{A}(\lambda)=\varnothing$, and $\mathcal{A}(a x)=\{a\} \cup \mathcal{A}(x)$ for each $a \in \Sigma$ and $x \in \Sigma^{\star}$. This mapping is extended to languages $L$ over $\Sigma$ by $\mathcal{A}(L)=\bigcup\{\mathcal{A}(w) \mid w \in L\}$.

Remember that a $\lambda$-free context-free grammar $G=(V, \Sigma, P, S)$ is in Chomsky normal form (CNF) if $P \subseteq N \times(N-\{S\})^{2} \cup N \times \Sigma$ where $N=V-\Sigma$. And $G$ is in Greibach normal form (GNF) if $P \subseteq N \times \Sigma(N-\{S\})^{\star}$. Particularly, $G$ is in Greibach $k$-form if $P \subseteq N \times \Sigma\left(\bigcup_{i=0}^{k}(N-\{S\})^{i}\right)$.

For a context-free grammar $G=(V, \Sigma, P, S)$ with $\alpha \in V, L(G, \alpha)$ denotes the language defined by $L(G, \alpha)=\left\{w \in \Sigma^{\star} \mid \alpha \Rightarrow^{\star} w\right\}$. Thus for the language $L(G)$ generated by $G$, we have $L(G)=L(G, S)$. Notice that, if $G$ is in CNF or GNF, then $G$ has no useless symbols, $L(G, \alpha)$ is a nonempty language for each symbol $\alpha$ in $V$, and $L(G, \alpha)=\{\alpha\}$ for each $\alpha$ in $\Sigma$. 
In studying $C_{n}$ we need, as in [3], subwords of $a_{1} a_{2} \cdots a_{n} a_{1} \cdots a_{n-1}$; so we consider the set $F_{k}^{n}$ all subwords of length $k(1 \leq k \leq n)$ that obey the circular succession relation $\prec$ on $\Sigma_{n}$ defined by: $a_{i} \prec a_{j}$ if and only if either (i) $i<n$ and $j=i+1$ or (ii) $i=n$ and $j=1$; cf. $\S 6.1$ in [10]. Clearly, $\prec$ is not a transitive relation: it is a kind of successor relation. Then the formal definition of $F_{k}^{n}$ reads

$$
F_{k}^{n}=\left\{x \in \Sigma_{n}^{\star}\left|\exists u, v \in \Sigma_{n}^{\star}: u x v=a_{1} a_{2} \cdots a_{n} a_{1} a_{2} \cdots a_{k-1} ;\right| x \mid=k\right\}
$$

with $1 \leq k \leq n$; their partial unions $\mathcal{Q}_{m}^{n}$ are defined by $\mathcal{Q}_{m}^{n}=\bigcup_{k=1}^{m} F_{k}^{n}(1 \leq m \leq n)$.

For a finite set $X$, we denote the cardinality of $X$ by $\# X$. Then we obviously have $C_{n}=F_{n}^{n}=\mathcal{Q}_{n}^{n}-\mathcal{Q}_{n-1}^{n}, \# F_{k}^{n}=n(1 \leq k \leq n), \# \mathcal{Q}_{m}^{n}=m n$ and $\# C_{n}=\# F_{n}^{n}=n$.

\section{Elementary Properties}

We first recall some simple properties of grammars in GNF that generate $L_{n}$ (the language of all permutations over $\Sigma_{n}$ ). From [4] we quote the following results.

Proposition 3.1. For $n \geq 1$, let $G_{n}=\left(V_{n}, \Sigma_{n}, P_{n}, S_{n}\right)$ be a context-free grammar in GNF that generates $L_{n}$, and let $A, B \in N_{n}=V_{n}-\Sigma_{n}$.

(1) The language $L\left(G_{n}, A\right)$ is a nonempty subset of an isomorphic copy $M_{k}$ of the language $L_{k}$ for some $k(1 \leq k \leq n)$. Consequently, each string $z$ in $L\left(G_{n}, A\right)$ has length $k, z$ consists of $k$ different symbols, and $\mathcal{A}(z)=\mathcal{A}\left(L\left(G_{n}, A\right)\right)$.

(2) If $L\left(G_{n}, A\right) \cap L\left(G_{n}, B\right) \neq \varnothing$, then $\mathcal{A}\left(L\left(G_{n}, A\right)\right)=\mathcal{A}\left(L\left(G_{n}, B\right)\right)$.

(3) If $A \rightarrow a A_{1} A_{2} \cdots A_{m}$ is a rule in $G_{n}$, then for each $(i, j)$ with $1 \leq i<j \leq m$, $\mathcal{A}\left(L\left(G_{n}, A_{i}\right)\right) \cap \mathcal{A}\left(L\left(G_{n}, A_{j}\right)\right)=\varnothing, a \notin \mathcal{A}\left(L\left(G_{n}, A_{k}\right)\right)$ with $1 \leq k \leq m$, and

$$
\mathcal{A}\left(L\left(G_{n}, A\right)\right)=\{a\} \cup \mathcal{A}\left(L\left(G_{n}, A_{1}\right)\right) \cup \mathcal{A}\left(L\left(G_{n}, A_{2}\right)\right) \cup \cdots \cup \mathcal{A}\left(L\left(G_{n}, A_{m}\right)\right) .
$$

This result gives rise to the following equivalence relation on $N_{n}: A$ and $B$ in $N_{n}$ are called equivalent if $|x|=|y|$ for some $x \in L\left(G_{n}, A\right)$ and some $y \in L\left(G_{n}, B\right)$. The equivalence classes are denoted by $\left\{E_{n, k}\right\}_{k=1}^{n}$. The number of elements $\# E_{n, k}$ of the equivalence class $E_{n, k}$ will be denoted by $D(n, k)(1 \leq k \leq n)$.

Example 3.2. Consider $G_{3}^{P}=\left(V_{3}, \Sigma_{3}, P_{3}, A_{123}\right)$ with $N_{3}=\left\{A_{123}, A_{12}, A_{13}, A_{23}\right.$, $\left.A_{1}, A_{2}, A_{3}\right\}$ and $P_{3}$ consists of $A_{123} \rightarrow a_{1} A_{23}\left|a_{2} A_{13}\right| a_{3} A_{12}, A_{12} \rightarrow a_{1} A_{2} \mid a_{2} A_{1}$, $A_{13} \rightarrow a_{1} A_{3}\left|a_{3} A_{1}, A_{23} \rightarrow a_{2} A_{3}\right| a_{3} A_{2}, A_{1} \rightarrow a_{1}, A_{2} \rightarrow a_{2}$ and $A_{3} \rightarrow a_{3}$. Clearly, $G_{3}^{P}$ is in GNF. Then $L\left(G_{3}^{P}\right)=L_{3}, E_{3,3}=\left\{A_{123}\right\}, E_{3,2}=\left\{A_{12}, A_{13}, A_{23}\right\}, E_{3,1}=\left\{A_{1}, A_{2}, A_{3}\right\}$, and hence $D(3,3)=1$ and $D(3,2)=D(3,1)=3$.

Proposition 3.1 relies on the fact that each word in $L\left(G_{n}\right)$ is a permutation. As circular shifts are special permutations, Proposition 3.1 still applies; but what is particular about generating $C_{n}$ rather than $L_{n}$ is expressed in Proposition 3.4, the proof of which depends on the following result from [3].

Lemma 3.3. If $X$ is a nonempty proper subalphabet of $\Sigma_{n}$, then there exists at most one word $x$ with $\mathcal{A}(x)=X$ such that $x$ satisfies the circular succession relation. And if 
$X=\left\{b_{1}, b_{2}, \ldots, b_{l}\right\}$, then $x=b_{p(1)} b_{p(2)} \cdots b_{p(l)}$ provided there exists a permutation $p$ of $\{1,2, \ldots, l\}$ such that $b_{p(1)} \prec b_{p(2)} \prec \cdots \prec b_{p(l)}$.

For each $w \in \Sigma_{n}^{\star}$, let $\underline{\alpha}(w)$ be the first and $\underline{\omega}(w)$ be the last symbol of $w$. Thus if $w=\sigma_{1} \sigma_{2} \cdots \sigma_{m}$ with $\sigma_{i} \in \Sigma(1 \leq i \leq m)$, then $\underline{\alpha}(w)=\sigma_{1}$ and $\underline{\omega}(w)=\sigma_{m}$.

Proposition 3.4. Let $G_{n}=\left(V_{n}, \Sigma_{n}, P_{n}, S_{n}\right)$ be a context-free grammar in GNF that generates $C_{n}$, and let $\alpha, \beta \in V_{n}-\left\{S_{n}\right\}$.

(1) For each $\alpha$, the language $L\left(G_{n}, \alpha\right)$ is a singleton.

(2) If $L\left(G_{n}, \alpha\right) \cap L\left(G_{n}, \beta\right) \neq \varnothing$, then $L\left(G_{n}, \alpha\right)=L\left(G_{n}, \beta\right)$.

(3) If $A \rightarrow a A_{1} A_{2} \cdots A_{m}$ is in $P_{n}$ with $L\left(G_{n}, A_{i}\right)=\left\{x_{i}\right\}(1 \leq i \leq m)$, then $a \prec \underline{\alpha}\left(x_{1}\right)$ and for each $i(1 \leq i<m), \underline{\omega}\left(x_{i}\right) \prec \underline{\alpha}\left(x_{i+1}\right)$. Consequently, if $L\left(G_{n}, A_{i}\right)=\left\{x_{i}\right\} \subseteq \bar{F}_{\Lambda(i)}^{n}$, then $L\left(G_{n}, A\right)=\left\{a x_{1} x_{2} \cdots x_{m}\right\} \subseteq F_{k}^{n}$ with $k=1+\sum_{i=1}^{m} \Lambda(i)$.

Proof. (1) $G_{n}$ is in GNF; so each symbol $\alpha$ in $V_{n}-\left\{S_{n}\right\}$ is useful: there is a derivation $S_{n} \Rightarrow^{+} \varphi \alpha \psi \Rightarrow^{+} \varphi x_{\alpha} \psi \Rightarrow^{+} x$ where $x$ is a circular shift, $\varphi \psi \neq \lambda, \mathcal{A}(x)=\Sigma_{n}$, and $1 \leq \# \mathcal{A}\left(x_{\alpha}\right)<n$. Now, by Lemma 3.3, $L\left(G_{n}, \alpha\right)$ contains at most one word over $\Sigma_{n}$, and since $L\left(G_{n}, \alpha\right)$ is nonempty, $L\left(G_{n}, \alpha\right)$ is a singleton.

(2) As $L\left(G_{n}, \alpha\right)$ and $L\left(G_{n}, \beta\right)$ are singletons by $(1), L\left(G_{n}, \alpha\right) \cap L\left(G_{n}, \beta\right) \neq \varnothing$ implies that they are equal.

Finally, (3) is a direct consequence of the fact that $L\left(G_{n}, A\right) \subseteq \mathcal{Q}_{n}^{n}$.

Henceforth, in examples we will always assume tacitly that $E_{n, 1}=\left\{A_{1}, \ldots, A_{n}\right\}$ and we will use $R_{n}=\left\{A_{i} \rightarrow a_{i} \mid A_{i} \in E_{n, 1}\right\}$.

Example 3.5. Consider $G_{4}^{U}=\left(V_{4}, \Sigma_{4}, P_{4}, S_{4}\right)$ in GNF with $P_{4}=\left\{S_{4} \rightarrow a_{1} A_{23} A_{4} \mid\right.$ $\left.a_{2} A_{34} A_{1}\left|a_{3} A_{41} A_{2}\right| a_{4} A_{12} A_{3}, A_{12} \rightarrow a_{1} A_{2}, A_{23} \rightarrow a_{2} A_{3}, A_{34} \rightarrow a_{3} A_{4}, A_{41} \rightarrow a_{4} A_{1}\right\} \cup R_{4}$. Then $L\left(G_{4}^{U}\right)=C_{4}, E_{4,4}=\left\{S_{4}\right\}, E_{4,3}=\varnothing, E_{4,2}=\left\{A_{12}, A_{23}, A_{34}, A_{41}\right\}, E_{4,1}=$ $\left\{A_{1}, A_{2}, A_{3}, A_{4}\right\}, D(4,4)=1, D(4,3)=0$ and $D(4,2)=D(4,1)=4$. Since $S_{4} \rightarrow a_{3} A_{41} A_{2}$ is in $P_{4}$, we have $L\left(G_{4}, A_{41}\right)=\left\{a_{4} a_{1}\right\}, L\left(G_{4}, A_{2}\right)=\left\{a_{2}\right\}, a_{3} \prec a_{4}=\underline{\alpha}\left(a_{4} a_{1}\right)$ and $\underline{\omega}\left(a_{4} a_{1}\right)=a_{1} \prec a_{2}=\underline{\alpha}\left(a_{2}\right)$.

As measures for the descriptional complexity of $G_{n}$ from $\left\{G_{n}\right\}_{n \geq 1}$, we use $\nu(n)=\# N_{n}$ and $\pi(n)=\# P_{n}$; cf. [11, 13, 14, 7, 5, 1,6]. A less-known measure has been introduced in $[2,3]$; viz. the number of left-most derivations $\delta(n)$ of $G_{n}$. Remember that in a leftmost derivation the leftmost nonterminal is always expanded. Thus $\delta(n)=\#\left\{S_{n} \Rightarrow_{L}^{\star} x \mid x \in\right.$ $\left.L\left(G_{n}\right)\right\}$, where $\Rightarrow_{L}$ denotes the leftmost derivation relation. Clearly, this measure makes sense when we generate a finite language by a $\lambda$-free grammar with bounded ambiguity.

Notice that these descriptional complexity measures depend on $n$ as well as on the family under consideration; so we use $\nu_{\alpha}(n), \pi_{\alpha}(n)$ and $\delta_{\alpha}(n)$ in the context of a family $\left\{G_{n}^{\alpha}\right\}_{n \geq 1}$ of which the individual members are labeled by $\alpha$.

Example 3.6. For $G_{3}^{P}$ of Example 3.2, we have $\nu_{P}(3)=7, \pi_{P}(3)=12$ and $\delta_{P}(3)=3 !=6$, since $G_{3}^{P}$ is unambiguous [2]. Similarly, for the unambiguous $G_{4}^{U}$ of Example 3.5, we have $\nu_{U}(4)=9, \pi_{U}(4)=12$ and $\delta_{U}(4)=4$. 
For each family $\left\{G_{n}^{\alpha}\right\}_{n \geq 1}=\left\{\left(V_{n}, \Sigma_{n}, P_{n}, S_{n}\right)\right\}_{n \geq 1}$ for $\left\{C_{n}\right\}_{n \geq 1}$ to be considered in the sequel, we assume that the first two (unspecified) elements $G_{1}^{\alpha}$ and $G_{2}^{\alpha}$ satisfy

- $N_{1}=\left\{S_{1}\right\}$ and $P_{1}=\left\{S_{1} \rightarrow a_{1}\right\}$ for $G_{1}^{\alpha}$, and

- $N_{2}=\left\{S_{2}, A_{1}, A_{2}\right\}, P_{2}=\left\{S_{2} \rightarrow a_{1} A_{2} \mid a_{2} A_{1}, A_{1} \rightarrow a_{1}, A_{2} \rightarrow a_{2}\right\}$ for $G_{2}^{\alpha}$.

Then $\nu_{\alpha}(1)=\pi_{\alpha}(1)=\delta_{\alpha}(1)=1, \nu_{\alpha}(2)=3, \pi_{\alpha}(2)=4, \delta_{\alpha}(2)=2$, whereas for $n \geq 3$, $\nu_{\alpha}(n)=\sum_{k=1}^{n} D(n, k) \geq n+1, \pi_{\alpha}(n) \geq n+2$ and $\delta_{\alpha}(n) \geq n$. This implies that specifying a family $\left\{G_{n}^{\alpha}\right\}_{n \geq 1}$ reduces to defining the family $\left\{G_{n}^{\alpha}\right\}_{n \geq 3}$.

As an illustration we consider a simple family of grammars in GNF for $\left\{C_{n}\right\}_{n \geq 1}$. It is based on a single nonterminal $S_{n}$ and the trivial set of rules $\left\{S_{n} \rightarrow w \mid w \in C_{n}\right\}$. To obtain grammars in GNF we need isomorphisms $\varphi_{n}: \Sigma_{n} \rightarrow\left\{A_{1}, A_{2}, \ldots, A_{n}\right\}$ defined by $\varphi_{n}\left(a_{i}\right)=$ $A_{i}(1 \leq i \leq n)$, that are extended to words by $\varphi_{n}\left(\sigma_{1} \sigma_{2} \cdots \sigma_{k}\right)=\varphi_{n}\left(\sigma_{1}\right) \varphi_{n}\left(\sigma_{2}\right) \cdots \varphi_{n}\left(\sigma_{k}\right)$ $\left(\sigma_{i} \in \Sigma_{n}, 1 \leq i \leq k\right)$.

Definition 3.7. $\left\{G_{n}^{T}\right\}_{n \geq 1}$ is given by $\left\{\left(V_{n}, \Sigma_{n}, P_{n}, S_{n}\right)\right\}_{n \geq 1}$ with for $n \geq 3$,

- $N_{n}=V_{n}-\Sigma_{n}=\left\{S_{n}\right\} \cup\left\{A_{i} \mid 1 \leq i \leq n\right\}$,

- $P_{n}=\left\{S_{n} \rightarrow \sigma_{1} \varphi_{n}\left(\sigma_{2} \cdots \sigma_{n}\right) \mid \sigma_{1} \sigma_{2} \cdots \sigma_{n} \in C_{n}\right\} \cup\left\{A_{i} \rightarrow a_{i} \mid 1 \leq i \leq n\right\}$.

In the sequel we will slightly change our notation in order to reduce the number of subscript levels: if $x=a_{j} \cdots a_{k}$, we will write $A_{j \cdots k}$ for $A_{x}$ instead of $A_{a_{j} \cdots a_{k}}$; cf. Examples 3.2 and 3.5 above. In this way the set of indices $\{1,2, \ldots, n\}$ inherits the linear order of $\Sigma_{n}$; a similar remark applies with respect to the $\prec$-relation.

Example 3.8. For $n=3$, we have $G_{3}^{T}=\left(V_{3}, \Sigma_{3}, P_{3}, S_{3}\right)$ with $P_{3}^{T}=\left\{S_{3} \rightarrow a_{1} A_{2} A_{3} \mid\right.$ $\left.a_{2} A_{3} A_{1} \mid a_{3} A_{1} A_{2}\right\} \cup R_{3}$. Now $E_{3,3}=\left\{S_{3}\right\}, E_{3,2}=\varnothing, E_{3,1}=\left\{A_{1}, A_{2}, A_{3}\right\}, D(3,3)=1$, $D(3,2)=0, D(3,1)=3, \nu_{T}(3)=4, \pi_{T}(3)=6$ and $\delta_{T}(3)=3$.

The following result easily follows from Definition 3.7.

Proposition 3.9. For the family $\left\{G_{n}^{T}\right\}_{n \geq 1}$ of Definition 3.7 we have for $n \geq 3$,

(2) $\nu_{T}(n)=n+1$,

(3) $\pi_{T}(n)=2 n$,

(4) $\delta_{T}(n)=n$.

\section{Greibach 2-form - A Straightforward Approach}

The trivial family $\left\{G_{n}^{T}\right\}_{n \geq 1}$ of grammars in (unrestricted) GNF of Definition 3.7 gives rise to simple results that are not very interesting. Therefore we restrict ourselves in the sequel to grammars in Greibach $k$-form with $k=1,2$. It turns out that in those cases the corresponding descriptional complexity measures are less trivial.

The idea on which our next family of grammars is based stems from Proposition 3.4: we have nonterminals $A_{x}$ for all strings $x$ in $\mathcal{Q}_{n-1}^{n}$ with $|x|=k<n$ such that $L\left(G_{n}, A_{x}\right)=\{x\}$ 
and $A_{x} \in E_{n, k}$. For the words in $C_{n}=\mathcal{Q}_{n}^{n}-\mathcal{Q}_{n-1}^{n}$ we have rules $S_{n} \rightarrow a A_{x} A_{y}$ for all nonempty words $x$ and $y$ with axy $\in C_{n}$ and $E_{n, n}=\left\{S_{n}\right\}$.

Definition 4.1. $\left\{G_{n}^{0}\right\}_{n \geq 1}$ is given by $\left\{\left(V_{n}, \Sigma_{n}, P_{n}, S_{n}\right)\right\}_{n \geq 1}$ with for $n \geq 3$,

- $N_{n}=V_{n}-\Sigma_{n}=\left\{S_{n}\right\} \cup\left\{A_{x} \mid x \in \mathcal{Q}_{n-2}^{n}\right\}$,

- $P_{n}=\left\{S_{n} \rightarrow a A_{x} A_{y} \mid a x y \in C_{n} ; a \in \Sigma_{n} ; x, y \in \Sigma_{n}^{+}\right\} \cup\left\{A_{a} \rightarrow a \mid a \in \Sigma_{n}\right\} \cup$

$$
\begin{aligned}
& \cup\left\{A_{a x y} \rightarrow a A_{x} A_{y} \mid a \in \Sigma_{n} ; a x y \in \mathcal{Q}_{n-1}^{n} ; x, y \in \Sigma_{n}^{+}\right\} \cup \\
& \cup\left\{A_{a b} \rightarrow a A_{b} \mid a, b \in \Sigma_{n} ; a \prec b\right\} .
\end{aligned}
$$

Example 4.2. Consider $G_{5}^{0}=\left(V_{5}, \Sigma_{5}, P_{5}, S_{5}\right)$ with $P_{5}=\left\{S_{5} \rightarrow a_{1} A_{2} A_{345}\left|a_{1} A_{23} A_{45}\right|\right.$ $a_{1} A_{234} A_{5}\left|a_{2} A_{3} A_{451}\right| a_{2} A_{34} A_{51}\left|a_{2} A_{345} A_{1}\right| a_{3} A_{4} A_{512}\left|a_{3} A_{45} A_{12}\right| a_{3} A_{451} A_{2}\left|a_{4} A_{5} A_{123}\right|$ \begin{tabular}{l|l|l|l|l}
$a_{4} A_{51} A_{23}$ & $a_{4} A_{512} A_{3}$ & $a_{5} A_{1} A_{234}$ & $a_{5} A_{12} A_{34}$ & $a_{5} A_{123} A_{4}, A_{123}$
\end{tabular}$\rightarrow a_{1} A_{2} A_{3}, A_{234} \rightarrow$ $a_{2} A_{3} A_{4}, A_{345} \rightarrow a_{3} A_{4} A_{5}, A_{451} \rightarrow a_{4} A_{5} A_{1}, A_{512} \rightarrow a_{5} A_{1} A_{2}, A_{12} \rightarrow a_{1} A_{2}, A_{23} \rightarrow$ $\left.a_{2} A_{3}, A_{34} \rightarrow a_{3} A_{4}, A_{45} \rightarrow a_{4} A_{5}, A_{51} \rightarrow a_{5} A_{1}\right\} \cup R_{5}$.

We have $E_{5,5}=\left\{S_{5}\right\}, E_{5,4}=\varnothing, E_{5,3}=\left\{A_{123}, A_{234}, A_{345}, A_{451}, A_{512}\right\}, E_{5,2}=$ $\left\{A_{12}, A_{23}, A_{34}, A_{45}, A_{51}\right\}, E_{5,1}=\left\{A_{1}, A_{2}, A_{3}, A_{4}, A_{5}\right\}, D(5,5)=1, D(5,4)=0, D(5,3)=$ $D(5,2)=D(5,1)=5$. Consequently, $\nu_{0}(5)=16, \pi_{0}(5)=30$ and $\delta_{0}(5)=15$; hence $G_{5}^{0}$ is ambiguous.

In general, if $S$ is a statement that can be true or false, then $[S]$ is equal to 1 if $S$ is true, and to 0 otherwise; cf. [10].

Proposition 4.3. For the family $\left\{G_{n}^{0}\right\}_{n \geq 1}$ of Definition 4.1 we have for $n \geq 3$,

(2) $\nu_{0}(n)=n^{2}-2 n+1$,

(3) $\pi_{0}(n)=[n \geq 4] \cdot\left(\frac{1}{2} n^{3}-\frac{7}{2} n^{2}+6 n\right)+n^{2}$,

(4) $\delta_{0}(n)=n^{2}-2 n$.

Proof. From Definition 4.1 it follows that for $n \geq 3, \nu_{0}(n)=\# N_{n}=1+\# \mathcal{Q}_{n-2}^{n}=$ $1+(n-2) n=n^{2}-2 n+1$, while $\pi_{0}(n)=h_{0}(n)+h_{1}(n)+h_{2}(n)+h_{3}(n)$ with

$$
\begin{aligned}
& h_{0}(n)=\#\left\{S_{n} \rightarrow a A_{x} A_{y} \mid a x y \in C_{n} ; a \in \Sigma_{n} ; x, y \in \Sigma_{n}^{+}\right\}, \\
& h_{1}(n)=\#\left\{A_{a x y} \rightarrow a A_{x} A_{y} \mid a \in \Sigma_{n} ; a x y \in \mathcal{Q}_{n-1}^{n} ; x, y \in \Sigma_{n}^{+}\right\}, \\
& h_{2}(n)=\#\left\{A_{a b} \rightarrow a A_{b} \mid a, b \in \Sigma_{n} ; a \prec b\right\}, \\
& h_{3}(n)=\#\left\{A_{a} \rightarrow a \mid a \in \Sigma_{n}\right\} .
\end{aligned}
$$

Clearly, $h_{0}(n)=n(n-2)$ and $h_{2}(n)=h_{3}(n)=n$. For $h_{1}$ we observe that $h_{1}(3)=0$, and for $n \geq 4$, we have $h_{1}(n)=\sum_{k=3}^{n-2}(k-2) \cdot n=\frac{1}{2} n(n-3)(n-4)=\frac{1}{2} n^{3}-\frac{7}{2} n^{2}+6 n$. So $\pi_{0}(n)=n(n-2)+[n \geq 4] \cdot\left(\frac{1}{2} n^{3}-\frac{7}{2} n^{2}+6 n\right)+n+n=[n \geq 4] \cdot\left(\frac{1}{2} n^{3}-\frac{7}{2} n^{2}+6 n\right)+n^{2}$.

The grammar $G_{n}^{0}$ generates $n$ strings, each of which can be obtained by a left-most derivation in $n-2$ ways $(n \geq 3)$; consequently, we have $\delta_{0}(n)=n(n-2)$. 


\section{Greibach 1-form - An Improvement}

The next grammar family $\left\{G_{n}^{1}\right\}_{n \geq 1}$ to generate $\left\{C_{n}\right\}_{n \geq 1}$ consists of context-free grammars in Greibach 1-form; this family is closely related to Definition 5.1 in [3] which in turn has been inspired by generating $\left\{C_{n}\right\}_{n \geq 1}$ with regular grammars.

Definition 5.1. $\left\{G_{n}^{1}\right\}_{n \geq 1}$ is given by $\left\{\left(V_{n}, \Sigma_{n}, P_{n}, S_{n}\right)\right\}_{n \geq 1}$ with for $n \geq 3$,

- $N_{n}=V_{n}-\Sigma_{n}=\left\{S_{n}\right\} \cup\left\{A_{x} \mid x \in \mathcal{Q}_{n-1}^{n}\right\}$,

- $P_{n}=\left\{S_{n} \rightarrow a A_{x} \mid a x \in C_{n} ; a \in \Sigma_{n}\right\} \cup\left\{A_{a} \rightarrow a \mid a \in \Sigma_{n}\right\} \cup$

$$
\cup\left\{A_{a x} \rightarrow a A_{x} \mid a x \in \mathcal{Q}_{n-1}^{n} ; a \in \Sigma_{n}, x \in \Sigma_{n}^{+}\right\} .
$$

Example 5.2. For $n=3$, we obtain $G_{3}^{1}=\left(V_{3}, \Sigma_{3}, P_{3}, S_{3}\right)$ with $P_{3}=\left\{S_{3} \rightarrow a_{1} A_{23} \mid\right.$ $\left.a_{2} A_{31} \mid a_{3} A_{12}, A_{12} \rightarrow a_{1} A_{2}, A_{23} \rightarrow a_{2} A_{3}, A_{31} \rightarrow a_{3} A_{1}\right\} \cup R_{3}$

Then we have $E_{3,3}=\left\{S_{3}\right\}, E_{3,2}=\left\{A_{12}, A_{23}, A_{31}\right\}, E_{3,1}=\left\{A_{1}, A_{2}, A_{3}\right\}, D(3,3)=1$, $D(3,2)=D(3,1)=3, \nu_{1}(3)=7, \pi_{1}(3)=9$ and $\delta_{1}(3)=3$.

The proof of the following result is similar to the one of Proposition 5.3 in [3].

Proposition 5.3. For the family $\left\{G_{n}^{1}\right\}_{n \geq 1}$ of Definition 5.1 we have for $n \geq 3$,

(2) $\nu_{1}(n)=n^{2}-n+1$,

(3) $\pi_{1}(n)=n^{2}$,

(4) $\delta_{1}(n)=n$.

Comparing Propositions 4.3 and 5.3 yields for $n \geq 4, \nu_{0}(n)<\nu_{1}(n), \pi_{0}(n)>\pi_{1}(n)$ and $\delta_{0}(n)>\delta_{1}(n)$. The latter two inequalities may be considered as an improvement; the price we have to pay is $n$ additional nonterminal symbols.

\section{Families of Unambiguous Grammars}

In [3] we argued that a first step towards minimal grammars in CNF is to avoid ambiguity. The situation for the GNF is very similar: the following crucial result and its proof are almost identical to the one for $\mathrm{CNF}$ in [3].

Proposition 6.1. Let $\left\{G_{n}\right\}_{n \geq 1}$ be a family of grammars in GNF that generates $\left\{C_{n}\right\}_{n \geq 1}$. Then $\delta(n)=n$ if and only if $\pi(n)=\nu(n)+n-1$.

The proof tells us that in an unambiguous grammar for $C_{n}$, there are $n$ rules for $S_{n}$ and a single rule for each $A \in N_{n}-\left\{S_{n}\right\}$. So we try to minimize $\nu(n)$, and as a consequence $\pi(n)$ will reach its minimum value as well. Clearly, Proposition 6.1 applies to $\left\{G_{n}^{1}\right\}_{n \geq 1}$ but not to $\left\{G_{n}^{0}\right\}_{n \geq 1}$. However, $\left\{G_{n}^{1}\right\}_{n \geq 1}$ is not the only family satisfying Proposition 6.1; another one will be introduced now.

Definition 6.2. $\left\{G_{n}^{2}\right\}_{n \geq 1}$ is given by $\left\{\left(V_{n}, \Sigma_{n}, P_{n}, S_{n}\right)\right\}_{n \geq 1}$ with for $n \geq 3$,

- $N_{n}=V_{n}-\Sigma_{n}=\left\{S_{n}\right\} \cup\left\{A_{a} \mid a \in \Sigma_{n}\right\} \cup M_{n}$ with for $m \geq 2$, 


$$
\begin{aligned}
& M_{2 m}=\left\{A_{x} \mid x \in F_{2 m-2}^{2 m} \cup F_{2 m-4}^{2 m} \cup \cdots \cup F_{2}^{2 m}\right\}, \text { and } \\
& M_{2 m-1}=\left\{A_{x} \mid x \in F_{2 m-3}^{2 m-1} \cup F_{2 m-5}^{2 m-1} \cup \cdots \cup F_{3}^{2 m-1}\right\}, \\
& \text { - } P_{n}=\left\{S_{n} \rightarrow a A_{b} A_{x} \mid a, b \in \Sigma_{n} ; x \in \Sigma_{n}^{+} ; a b x \in C_{n} ; x \in F_{n-2}^{n}\right\} \cup Q_{n} \cup \\
& \cup\left\{A_{a b x} \rightarrow a A_{b} A_{x} \mid a, b \in \Sigma_{n} ; A_{a b x} \in M_{n} ; x \in \Sigma_{n}^{+}\right\} \cup\left\{A_{a} \rightarrow a \mid a \in \Sigma_{n}\right\}
\end{aligned}
$$

with for $m \geq 2, Q_{2 m}=\left\{A_{a b} \rightarrow a A_{b} \mid a, b \in \Sigma_{n} ; a \prec b\right\}$, and $Q_{2 m-1}=\varnothing$.

Example 6.3. Let $G_{6}^{2}=\left(V_{6}, \Sigma_{6}, P_{6}, S_{6}\right)$ with $P_{6}=\left\{S_{6} \rightarrow a_{1} A_{2} A_{3456}\left|a_{2} A_{3} A_{4561}\right|\right.$ $a_{3} A_{4} A_{5612}\left|a_{4} A_{5} A_{6123}\right| a_{5} A_{6} A_{1234} \mid a_{6} A_{1} A_{2345}, A_{1234} \rightarrow a_{1} A_{2} A_{34}, A_{2345} \rightarrow a_{2} A_{3} A_{45}$, $A_{3456} \rightarrow a_{3} A_{4} A_{56}, A_{4561} \rightarrow a_{4} A_{5} A_{61}, A_{5612} \rightarrow a_{5} A_{6} A_{12}, A_{6123} \rightarrow a_{6} A_{1} A_{23}, A_{12} \rightarrow a_{1} A_{2}$, $\left.A_{23} \rightarrow a_{2} A_{3}, A_{34} \rightarrow a_{3} A_{4}, A_{45} \rightarrow a_{4} A_{5}, A_{56} \rightarrow a_{5} A_{6}, A_{61} \rightarrow a_{6} A_{1}\right\} \cup R_{6}$

Now $E_{6,6}=\left\{S_{n}\right\}, E_{6,5}=\varnothing, E_{6,4}=\left\{A_{1234}, A_{2345}, A_{3456}, A_{4561}, A_{5612}, A_{6123}\right\}, E_{6,3}=\varnothing$, $E_{6,2}=\left\{A_{12}, A_{23}, A_{34}, A_{45}, A_{56}, A_{61}\right\}$ and $E_{6,1}=\left\{A_{1}, A_{2}, A_{3}, A_{4}, A_{5}, A_{6}\right\}$. Then we obtain $\nu_{2}(6)=19<31=\nu_{1}(6)$ and $\pi_{2}(6)=24<36=\pi_{1}(6)$.

Proposition 6.4. For the family $\left\{G_{n}^{2}\right\}_{n \geq 1}$ of Definition 6.2, we have

$$
\begin{aligned}
& \text { (1) } D(n, n)=1, D(n, n-1)=0, D(n, 1)=n \text {, and for } 2 \leq k<n-1, \\
& D(n, k)=\text { if } k \equiv n \quad(\bmod 2) \text { then } n \text { else } 0, \\
& \text { (2) for } n \geq 3, \nu_{2}(n)=\frac{1}{2} n^{2}+\frac{1}{2} n \cdot[n \text { is odd }]+1, \\
& \text { (3) for } n \geq 3, \pi_{2}(n)=\frac{1}{2} n^{2}+\frac{1}{2} n \cdot([n \text { is odd }]+2), \\
& \text { (4) } \delta_{2}(n)=n \text {. }
\end{aligned}
$$

Proof. From Definition 6.2, Proposition 6.4(1) and (4) easily follow. Then for even $n$ with $n \geq 4$, we have $\nu_{2}(n)=1+2 n+\sum_{k=4}^{n-2} D(n, k)=1+2 n+\frac{1}{2}(n-4) n=\frac{1}{2} n^{2}+1$. For odd $n$ with $n \geq 3$, we obtain $\nu_{2}(n)=1+2 n+\sum_{k=3}^{n-2} D(n, k)=1+2 n+\frac{1}{2}(n-3) n=\frac{1}{2} n^{2}+\frac{1}{2} n+1$. Combining these two cases results in $\nu_{2}(n)=\frac{1}{2} n^{2}+\frac{1}{2} n \cdot[n$ is odd $]+1$ for $n \geq 3$. Finally, Proposition 6.1 implies Proposition 6.4(3).

From Propositions 5.3 and 6.4 it follows that for $n \geq 4$, we have $\nu_{2}(n)<\nu_{1}(n)$ and, consequently, $\pi_{2}(n)<\pi_{1}(n)$.

It is possible to continue in this way by introducing families $\left\{G_{n}^{k}\right\}_{n \geq 1}(k \geq 3)$ such that for each rule $A \rightarrow a B C, L\left(G_{n}^{k}, B\right)$ consists of a single word of length $k-1$. As in [3] the objections are twofold: the definitions become more complicated as $k$ increases, and we are probably left with $\nu_{k}(n)$ and $\pi_{k}(n)$ being functions in $\Theta\left(n^{2}\right)$.

\section{Towards a Family of Minimal Grammars}

For the CNF we defined in [3] a family of grammars $\left\{G_{n}^{k}\right\}_{n \geq 1}$ to be minimal if each $G_{k}$ is unambiguous and $\nu(n) \in \Theta(n)$; cf. Proposition 6.1. As we will see, this latter condition is likely to be too ambitious for the GNF. In [3] we also established the existence of a minimal family for the CNF; it turns out that the corresponding problem for the GNF remains open. But let us first have a look at a GNF-family as simple as the minimal CNF-family of [3]. 
Definition 7.1. $\left\{G_{n}^{\diamond}\right\}_{n \geq 1}$ is given by $\left\{\left(V_{n}, \Sigma_{n}, P_{n}, S_{n}\right)\right\}_{n \geq 1}$ with for even $n \geq 4$,

- $N_{n}=V_{n}-\Sigma_{n}=\left\{S_{n}, A_{1}, A_{2}, \ldots, A_{n}\right\} \cup\left\{A_{i \cdots k} \mid i=1,3,5, \ldots, n-1\right.$;

$$
\left.a_{i} \cdots a_{k} \in F_{n-2} \cup F_{n-4} \cup F_{n-6} \cup \cdots \cup F_{2}\right\},
$$

- $P_{n}=\left\{S_{n} \rightarrow a_{i} A_{j} A_{k \cdots m}\left|a_{j} A_{k \cdots m} A_{i}\right| i=1,3, \ldots, n-1 ; a_{i} a_{j} a_{k} \cdots a_{m} \in C_{n}\right\} \cup$

$$
\begin{aligned}
& \left\{A_{k} \rightarrow a_{k} \mid 1 \leq k \leq n\right\} \cup\left\{A_{i \ldots m} \rightarrow a_{i} A_{j} A_{k \cdots m} \mid i=1,3, \ldots, n-1 ;\right. \\
& \left.a_{i} a_{j} a_{k} \cdots a_{m} \in F_{n-2} \cup F_{n-4} \cup F_{n-6} \cup \cdots \cup F_{2}\right\},
\end{aligned}
$$

where $A_{k \ldots m}$ is taken equal to $\lambda$ whenever $a_{k} \cdots a_{m}$ equals $\lambda$;

and for odd $n \geq 3$,

- $N_{n}=V_{n}-\Sigma_{n}=\left\{S_{n}, A_{1}, A_{2}, \ldots, A_{n}\right\} \cup\left\{A_{i \cdots k} \mid i=1,3,5, \ldots, n\right.$;

$$
\left.a_{i} \cdots a_{k} \in F_{n-2} \cup F_{n-4} \cup F_{n-6} \cup \cdots \cup F_{3}\right\} \text {, }
$$

- $P_{n}=\left\{S_{n} \rightarrow a_{i} A_{j} A_{k \cdots m}\left|a_{j} A_{k \cdots m} A_{i}\right| a_{n} A_{12 \ldots(n-2)} A_{n-1} \mid i=1,3, \ldots, n-2\right.$;

$$
\begin{aligned}
& \left.a_{i} a_{j} a_{k} \cdots a_{m} \in C_{n}\right\} \cup\left\{A_{k} \rightarrow a_{k} \mid 1 \leq k \leq n\right\} \cup\left\{A_{i \ldots m} \rightarrow a_{i} A_{j} A_{k \cdots m} \mid\right. \\
& \left.i=1,3, \ldots, n-2 ; a_{i} a_{j} a_{k} \cdots a_{m} \in F_{n-2} \cup F_{n-4} \cup F_{n-6} \cup \cdots \cup F_{3}\right\} \cup \\
& \left\{A_{n 12 \ldots k} \rightarrow a_{n} A_{12 \ldots(k-1)} A_{k} \mid k=2,4, \ldots, n-3 ; a_{n} a_{1} \cdots a_{k} \in F_{n-2} \cup\right. \\
& \left.F_{n-4} \cup F_{n-6} \cup \cdots \cup F_{3}\right\} .
\end{aligned}
$$

Example 7.2. Let $G_{7}^{\diamond}=\left(V_{7}, \Sigma_{7}, P_{7}, S_{7}\right)$ with $P_{7}=\left\{S_{7} \rightarrow a_{1} A_{2} A_{34567}\left|a_{2} A_{34567} A_{1}\right|\right.$ $a_{3} A_{4} A_{56712}\left|a_{4} A_{56712} A_{3}\right| a_{5} A_{6} A_{71234}\left|a_{6} A_{71234} A_{5}\right| a_{7} A_{12345} A_{6}, A_{12345} \rightarrow a_{1} A_{2} A_{345}$, $A_{34567} \rightarrow a_{3} A_{4} A_{567}, A_{56712} \rightarrow a_{5} A_{6} A_{712}, A_{71234} \rightarrow a_{7} A_{123} A_{4}, A_{123} \rightarrow a_{1} A_{2} A_{3}, A_{345} \rightarrow$ $\left.a_{3} A_{4} A_{5}, A_{567} \rightarrow a_{5} A_{6} A_{7}, A_{712} \rightarrow a_{7} A_{1} A_{2}\right\} \cup R_{7}$.

Then $\nu_{\diamond}(7)=16<29=\nu_{2}(7), \pi_{\diamond}(7)=22<35=\pi_{2}(7)$ and $\delta_{\diamond}(7)=7$.

Proposition 7.3. For the family $\left\{G_{n}^{\diamond}\right\}_{n \geq 1}$ of Definition 7.1 we have

$D(n, n)=1, D(n, 1)=n$, and

for even $n$ and $k=2,4, \ldots, n-2, \quad D(n, k)=\frac{1}{2} n$,

for odd $n$ and $k=3,5, \ldots, n-2, \quad D(n, k)=\left\lceil\frac{1}{2} n\right\rceil$,

(2) $\nu_{\diamond}(n)=\frac{1}{4} n^{2}+\frac{1}{2} n+\frac{1}{4}+\frac{3}{4} \cdot[n$ is even $]$,

(3) $\pi_{\diamond}(n)=\frac{1}{4} n^{2}+\frac{3}{2} n-\frac{3}{4} \cdot[n$ is odd $]$,

(4) $\delta_{\diamond}(n)=n$.

Proof. It is easy to establish (1) and (4); then for even $n$ we have

$$
\nu_{\diamond}(n)=1+n+\left(\frac{1}{2} n-1\right) \frac{1}{2} n=\frac{1}{4} n^{2}+\frac{1}{2} n+1,
$$

and for odd $n$,

$$
\nu_{\diamond}(n)=1+n+\left\lfloor\frac{1}{2} n-1\right\rfloor\left\lceil\frac{1}{2} n\right\rceil=\frac{1}{4} n^{2}+\frac{1}{2} n+\frac{1}{4} .
$$

Finally, (3) follows from (2), (4) and Proposition 6.1.

Although this is an improvement with respect to Propositions $4.3,5.3$ and $6.4,\left\{G_{n}^{\diamond}\right\}_{n \geq 1}$ is by no means a minimal family as we will see from the following "divide-and-conquer" family; cf. $\S 8$ in [2]. 


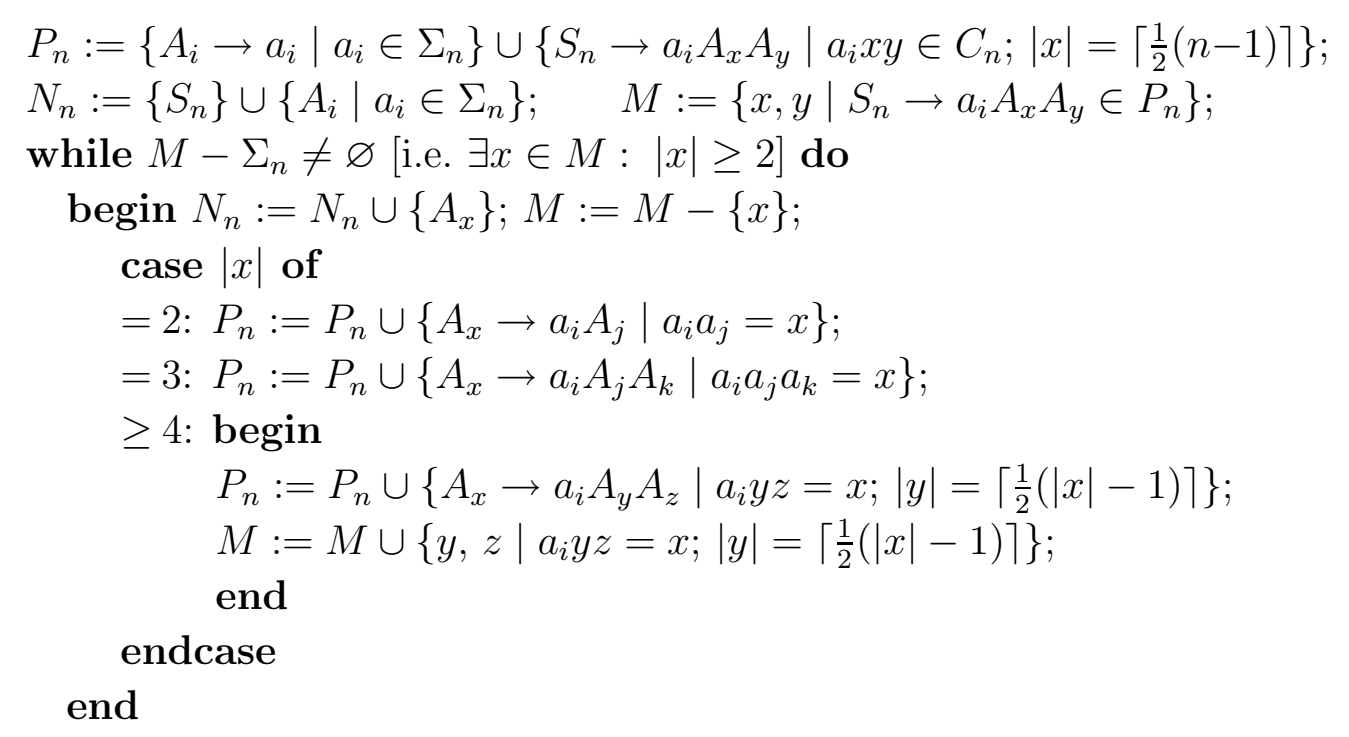

Figure 1: Algorithm to determine $N_{n}$ and $P_{n}$ of $G_{n}^{\bullet}$.

Definition 7.4. $\left\{G_{n}^{\bullet}\right\}_{n \geq 1}$ is given by $\left\{\left(V_{n}, \Sigma_{n}, P_{n}, S_{n}\right)\right\}_{n \geq 1}$ where the sets $N_{n}$ and $P_{n}$ are determined by the algorithm in Figure 1.

Example 7.5. $\quad G_{7}^{\bullet}=\left(V_{7}, \Sigma_{7}, P_{7}, S_{7}\right)$ with $P_{7}=\left\{S_{7} \rightarrow a_{1} A_{234} A_{567}\left|a_{2} A_{345} A_{671}\right|\right.$ \begin{tabular}{l|l|l|l|l}
$a_{3} A_{456} A_{712}$ & $a_{4} A_{567} A_{123}$ & $a_{5} A_{671} A_{234}$ & $a_{6} A_{712} A_{345}$ & $a_{7} A_{123} A_{456}, A_{123} \rightarrow a_{1} A_{2} A_{3}, A_{234} \rightarrow$
\end{tabular} $a_{2} A_{3} A_{4}, A_{345} \rightarrow a_{3} A_{4} A_{5}, A_{456} \rightarrow a_{4} A_{5} A_{6}, A_{567} \rightarrow a_{5} A_{6} A_{7}, A_{671} \rightarrow a_{6} A_{7} A_{1}, A_{712} \rightarrow$ $\left.a_{7} A_{1} A_{2}\right\} \cup R_{7}$. Now $\nu_{\bullet}(7)=15<16=\nu_{\diamond}(7), \pi_{\bullet}(7)=21<22=\nu_{\diamond}(7)$ and $\delta_{\bullet}(7)=7$.

\begin{tabular}{|c||r|r|r|r|r|r|r|r|r|r|r|r|r|r|}
\hline$n$ & 3 & 4 & 5 & 6 & 7 & 8 & 9 & 10 & 11 & 12 & 13 & 14 & 15 & 16 \\
\hline \hline$\nu_{\bullet}(n)$ & 4 & 9 & 11 & 19 & 15 & 33 & 28 & 41 & 34 & 61 & 53 & 71 & 46 & 96 \\
\hline$\pi_{\bullet}(n)$ & 6 & 12 & 15 & 24 & 21 & 40 & 36 & 50 & 44 & 72 & 65 & 84 & 60 & 111 \\
\hline
\end{tabular}

Table 1: $\nu_{\bullet}(n)$ and $\pi_{\bullet}(n)$ for $3 \leq n \leq 16$.

As usual in analyzing such a divide-and-conquer approach, a closed form for $\nu_{\bullet}(n)$ and $\pi_{\bullet}(n)$ is very hard or even impossible to obtain; for small values we refer to Table 1 . Only for special values of $n$ we can infer some manageable expressions.

Proposition 7.6. For the family $\left\{G_{n}^{\bullet}\right\}_{n \geq 1}$ we have in case $n=2^{k}-1(k \geq 2)$,

(1) $D(n, n)=1, D\left(n, 2^{i}-1\right)=n(i=1,2, \ldots, k-1)$, and $D(n, i)=0$ otherwise,

(2) $\nu_{\bullet}(n)=n \cdot \log _{2}(n+1)-n+1$,

(3) $\pi_{\bullet}(n)=n \cdot \log _{2}(n+1)$,

(4) $\delta_{\bullet}(n)=n$. 
So using this divide-and-conquer approach we end up with $\nu_{\bullet}(n)$ and $\pi_{\bullet}(n)$ in $\Theta(n$. $\left.\log _{2} n\right)$, rather than in $\Theta\left(n^{2}\right)$ as for the previous families.

\section{Concluding Remarks}

We discussed a few ways of generating the languages $\left\{C_{n}\right\}_{n \geq 1}$ of circular shifts by contextfree grammars $\left\{G_{n}\right\}_{n \geq 1}$ in GNF, and we compared these families with respect to the measures $\nu, \pi$ and $\delta$. Our results give rise to the following observation.

Conjecture 8.1. Any family of context-free grammars in $G N F\left\{G_{n}\right\}_{n \geq 1}$ that generates $\left\{C_{n}\right\}_{n \geq 1}$ must have measures $\nu(n)$ and $\pi(n)$ that are not bounded by any linear function in $n$.

The situation in the GNF-case differs considerably from the CNF-case: in [3] we established the existence of a minimal family in CNF for which $\nu$ and $\pi$ are linear functions in $n$ (even with small coefficients). For the GNF the definition of minimality remains a problem; viz. setting " $\left\{G_{n}\right\}_{n \geq 1}$ in GNF is minimal for $\left\{C_{n}\right\}_{n \geq 1}$ if (i) each $G_{n}$ is unambiguous, and (ii) $\nu(n) \in \Theta(f(n))$ " leaves us with the question of an adequate choice for $f(n)$. Conjecture 8.1 implies $f(n) \in \omega(n)$.

Taking $f(n)$ equal to $n \cdot \log _{2} n$ results in the minimality of $\left\{G_{n}^{\bullet}\right\}_{n \geq 1}$ (Proposition 7.6), but the question whether this family is also minimal in an absolute sense (i.e., does there exists no family with $\nu(n) \in \Theta\left(n \cdot \log _{2} n\right)$ and $\nu(n)<n \cdot \log _{2}(n+1)-n+1$ for $n$ large enough with $n=2^{k}-1$ and $k \geq 2$ ?) remains an open problem as well.

\section{References}

1. B. Alspach, P. Eades \& G. Rose, A lower-bound for the number of productions for a certain class of languages, Discrete Appl. Math. 6 (1983) 109-115.

2. P.R.J. Asveld, Generating all permutations by context-free grammars in Chomsky normal form, Theoret. Comput. Sci. 354 (2006) 118-130.

3. P.R.J. Asveld, Generating all circular shifts by context-free grammars in Chomsky normal form, CTIT TR 05-23 (2005), ISSN 1381-3625, University of Twente, Enschede, the Netherlands; to appear in J. Autom., Lang. Combin.

4. P.R.J. Asveld, Generating all permutations by context-free grammars in Greibach normal form, (in preparation).

5. W. Bucher, A note on a problem in the theory of grammatical complexity, Theoret. Comput. Sci. 14 (1981) 337-344.

6. W. Bucher, H.A. Maurer \& K. Culik II, Context-free complexity of finite languages, Theoret. Comput. Sci. 28 (1984) 277-285.

7. W. Bucher, H.A. Maurer, K. Culik II \& D. Wotschke, Concise description of finite languages, Theoret. Comput. Sci. 14 (1981) 227-246. 
8. J. Dassow, On the circular closure of languages, EIK 15 (1979) 87-94.

9. K. Ellul, B. Krawetz, J. Shallit \& M.-w. Wang, Regular expressions: new results and open problems, J. Autom. Lang. Comb. 9 (2004) 233-256.

10. R.L. Graham, D.E. Knuth \& O. Patashnik, Concrete Mathematics (1989), AddisonWesley, Reading, MA.

11. J. Gruska, Some classifications of context-free languages, Inform. Contr. 14 (1969) $152-179$.

12. M.A. Harrison, Introduction to Formal Language Theory (1978), Addison-Wesley, Reading, MA.

13. V.A. Iljuškin, The complexity of the grammatical description of context-free languages, Dokl. Akad. Nauk SSSR 203 (1972) 1244-1245 / Soviet Math. Dokl. 13 (1972) 533-535.

14. A. Kelemenová, Complexity of normal form grammars, Theoret. Comput. Sci. 28 (1984) 299-314.

15. G. Satta, personal communication (2002). 